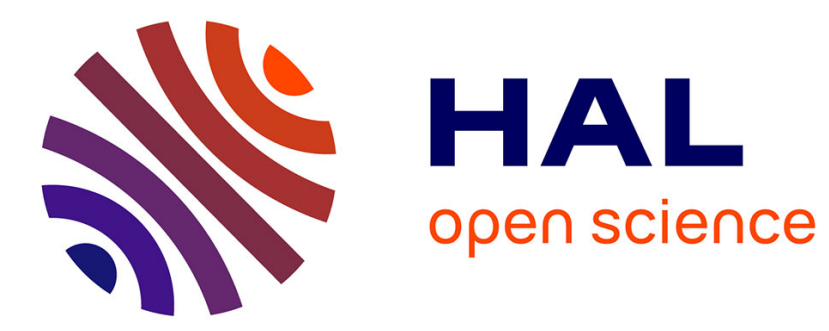

\title{
Evaluation du niveau de ploïdie des plantes d'une population de choux de Bruxelles (Brassica oleracea L. ssp. gemmifera) d'origine pollinique
}

Claire Doré, Jean-Charles Lescure, Florence Charlot

\section{- To cite this version:}

Claire Doré, Jean-Charles Lescure, Florence Charlot. Evaluation du niveau de ploïdie des plantes d'une population de choux de Bruxelles (Brassica oleracea L. ssp. gemmifera) d'origine pollinique. Agronomie, 1986, 6 (9), pp.797-801. hal-00884939

\section{HAL Id: hal-00884939 https://hal.science/hal-00884939}

Submitted on 1 Jan 1986

HAL is a multi-disciplinary open access archive for the deposit and dissemination of scientific research documents, whether they are published or not. The documents may come from teaching and research institutions in France or abroad, or from public or private research centers.
L'archive ouverte pluridisciplinaire HAL, est destinée au dépôt et à la diffusion de documents scientifiques de niveau recherche, publiés ou non, émanant des établissements d'enseignement et de recherche français ou étrangers, des laboratoires publics ou privés. 


\title{
Evaluation du niveau de ploïdie des plantes d'une population de choux de Bruxelles (Bras- sica oleracea L. ssp. gemmifera) d'origine polli- nique
}

Claire DORE
avec la collaboration technique de Jean-Charles LEScuRE \& Florence CHARIOT

I.N.R.A., Station de Génétique et d'Amélioration des Plantes, Centre de Recherches de Versailles, Route de Saint-Cyr, F 78000 Versailles

RÉSUMÉ

\begin{abstract}
L'obtention par culture d'anthères in vitro d'un nombre important de plantes nécessite de disposer d'une méthode rapide pour trier les plantes selon leur niveau de ploïdie.

Deux méthodes d'évaluation ont été comparées:

1. la mesure de la longueur des cellules de garde des stomates,

2. le comptage des chromosomes.

La mesure de la longueur des cellules de garde des stomates de l'épiderme inférieur des feuilles apparaît comme une intéressante alternative au comptage chromosomique.

Pour obtenir des résultats fiables, les mesures doivent, dans nos conditions, être effectuées sur 2 feuilles au moins de jeunes plantes en croissance.
\end{abstract}

Mots clés additionnels : Cutture d'anthères, niveau de ploidie, cellules de garde, stomate.

Ploidy level evaluation for a population of pollen plants of Brussels sprouts (Brassica oleracea $L$. ssp. gemmitera).

Obtaining numerous pollen plants of different levels of ploidy through anther culture has led us to look for a rapid method for sorting plants by ploidy level. Two methods of evaluation were compared : measuring the length of stomatal guard cells on the lower leaf epidermis and chromosome counting. The former could usefully replace the latter. To obtain reliable results, under our conditions, measures must be done on at least two leaves from young growing plants.

Additional key words : Anther culture, ploidy level, guard cell, stomata.

\section{INTRODUCTION}

La culture d'anthères de Brassica sp. fait l'objet de nombreux travaux à travers le monde. C'est sur le colza, Brassica napus L., que porte la majorité des résultats publiés. Dans tous les cas, les auteurs (KELLER \& ARMSTRONG, 1978 ; DUNWELl et al., 1985) mettent en évidence l'importance du génotype, des conditions de culture de la plante-mère et des séquences thermiques de la culture des anthères.

Ces facteurs apparaissent encore plus déterminants chez l'espèce Brassica oleracea L. où les sous-espèces italica (broccoli) et gemmifera (Chou de Bruxelles) ont été surtout étudiées (KELLER \& ARMSTRONG, 1983 ; ORTON \& BROWERS, 1985 ; LELU \& BOLlON, 1985).

Chez les Brassica, l'analyse cytologique montre que les plantes provenant de culture d'anthères ne sont pas toutes haploïdes ; elles peuvent être également diplö̈des, triploïdes ou même tétraploïdes (KELLER \& ARMSTRONG, 1977 ; GEORGE \& RAO, 1982).

Pour utiliser la culture d'anthères in vitro comme source de matériel homozygote dans un programme de sélection, il faut pouvoir disposer d'une technique simple et fiable permettant de connaître rapidement le niveau de ploïdie de chaque plante afin de séparer les 
haploïdes, dont il faut doubler le stock chromosomique, des diploïdes que l'on conserve et des triploïdes que l'on élimine.

Or, dès que le nombre de plantes devient important, le dénombrement chromosomique systématique est pratiquement irréalisable, surtout chez les Brassica dont les chromosomes sont petits. En outre, la possibilité d'observer des cellules en métaphase, stade favorable au dénombrement, dépend beaucoup du stade de développement des racines et de l'état physiologique de la plante.

Pour toutes ces raisons, nous avons recherché une alternative plus rapide mais au moins aussi fiable.

Parmi les diverses possibilités, nous avons retenu la mesure de la longueur des cellules de garde des stomates de l'épiderme des feuilles qui a été utilisée avec succès sur plusieurs espèces telles que Crepis zacintha (L.) Babc. (Y. Cauderon \& A. Cauderon, 1953), les Lolium sp. (SPECKMANN et al., 1965) et qui est déjà pratiquée sur le Chou de Bruxelles (OCKENDON et al., 1983).

Le présent article rapporte une adaptation de cette méthode dans nos conditions et définit les modalités techniques de son emploi. Sa fiabilité et son intérêt pratique sont évalués par rapport à la méthode de comptage chromosomique à l'aide d'un échantillon de 50 plantes. Son emploi est ensuite étendu à l'évaluation du niveau de ploïdie de l'ensemble des individus de la population d'origine pollinique.

\section{MATÉRIEL ET MÉTHODES}

\section{A. Matériel végétal}

La culture d'anthères in vitro d'une série de 22 plantes différentes, correspondant à une collection de variétés de Chou de Bruxelles cultivées par le Laboratoire d'Amélioration du Chou de la Station de Génétique et d'Amélioration des Plantes de Versailles, a permis d'obtenir, à partir de 7 plantes, environ 300 embryons d'origine pollinique. Parmi eux, une centaine a évolué en plantes viables après repiquage en terre à l'automne 1984

Ces plantes ont été cultivées en chambre climatisée en jours longs ( $16 \mathrm{~h})$ avec une alternance de température de $18{ }^{\circ} \mathrm{C}$ la nuit et $23{ }^{\circ} \mathrm{C}$ le jour.

\section{B. Techniques employées}

\section{Comptage chromosomique}

Le comptage chromosomique est effectué par la méthode de Feulgen sur des extrémités de racine en croissance active, selon lẹs modalités suivantes :

- prétraitement, dans une solution d'eau ordinaire saturée en $\alpha$ bromo-naphtalène, pendant $2 \mathrm{~h}$ à température ambiante,

- fixation dans l'alcool acétique, à $5{ }^{\circ} \mathrm{C}$ pendant $2 \mathrm{~h}$ au minimum,

- hydrolyse dans l'acide chlorhydrique $5 \mathrm{~N}$ pendant $30 \mathrm{mn}$ à température ambiante,
- coloration dans le réactif de Schiff pendant $2 \mathrm{~h}$

à température ambiante et à l'abri de la lumière,

- montage dans une goutte d'eau acétique,

- observation au microscope photonique.

\section{Mesure de la longueur des cellules de garde des sto- mates}

La technique consiste à prélever à l'aide d'une pince fine un fragment d'épiderme à la face inférieure du limbe d'une feuille. Ce fragment est monté dans une goutte d'eau entre lame et lamelle. L'ensemble est luté pour éviter un dessèchement rapide de la préparation.

L'observation est faite au microscope photonique équipé d'un micromètre oculaire afin d'estimer la longueur des cellules.

\section{RÉSULTATS}

\section{A. Choix d'un échantillon aussi homogène et repré- sentatif que possible de chaque plante}

\section{Etat des plantes}

Les $1^{\text {res }}$ observations ont montré qu'il est tout d'abord indispensable de se placer dans des conditions où les stomates sont bien turgescents, ce qui correspond évidemment à des conditions de bon éclairement et d'arrosage régulier.

\section{Montage de la préparation}

Il faut d'autre part effectuer le montage de la préparation immédiatement après prélèvement du fragment de feuille afin d'éviter son dessèchement qui provoque une altération des stomates.

\section{Age des feuilles}

Les feuilles très jeunes ne conviennent pas car les résultats obtenus sont, dans ce cas, trop peu discriminants d'un niveau de plö̈die à l'autre. De même, les feuilles âgées ne sauraient être utilisées car la dispersion des valeurs obtenues est trop grande (fig. 1). Pour avoir des résultats suffisamment homogènes pour une même feuille, nous avons retenu d'effectuer les mesures sur les feuilles adultes (c'est-à-dire complètement déployées) de jeunes plantes ayant 10 à 15 feuilles déjà dégagées du bourgeon terminal, ce qui correspond dans nos conditions à des plantes de 10 à $20 \mathrm{~cm}$ de hauteur.

\section{Nombre de mesures}

Entre la $4^{\mathrm{e}}$ et la $8^{\mathrm{e}}$ feuille dégagée du bourgeon principal (fig. 2), nous prélevons 2 feuilles, une de rang $\mathrm{n}$, l'autre de rang $\mathrm{n}+2$, pour faire 2 mesures de longueur des cellules de garde par feuille. Il faut en effet pouvoir détecter les divergences éventuelles entre les mesures de 2 feuilles d'une même plante. Il est important d'éviter de prélever un fragment au niveau d'une nervure car les stomates y ont un aspect différent. 




Figure 1

Corrélations entre les niveaux de ploidie observés par comptage chromosomique et la longueur des cellules de garde des stomates en fonction de la position de la feuille.

Equations des droites de régression:

Correlations between ploidy levels observed by chromosome counting and measurement of stomatal guard cell length according to the position of the leaf.

$1^{\text {re }}$ droite $=$

$\hat{y}_{1}=19,76+0,88 x$

avec $F=18$

$2^{e}$ droite $=$

$\hat{y}_{2}=16,34+0,69 x$

avec $F=140$

$3^{2}$ droite $=$

$\hat{y}_{3}=11,59+0,29 x$ avec $F=8,8$

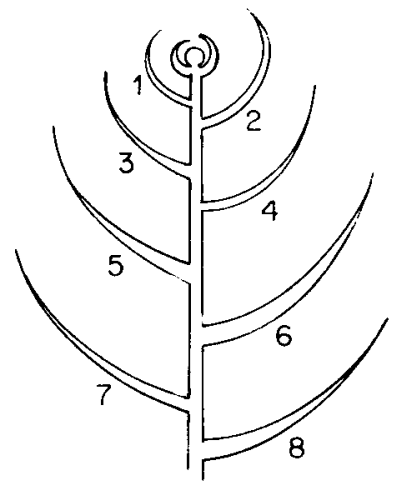

Figure 2

Coupe longitudinale axiale d'une jeune plante de Chou montrant la position des feuilles prélevées.

Axial longitudinal section of a young cabbage plant showing the position of the leaves used for stomatal study.

\section{Longueurs obtenues}

En tenant compte de ces éléments, il est possible de distinguer nettement 3 classes à partir des moyennes des longueurs des cellules de garde des stomates qui correspondent à 3 classes de plantes.

En effet, selon les plantes, les moyennes des mesures varient autour de $14 \mu \mathrm{m}$ (fig. 3), autour de $20 \mu \mathrm{m}$ (fig. 4) ou bien sont supérieures à $24 \mu \mathrm{m}$ (fig. 5).

\section{B. Evaluation des niveaux de ploïdie et comparaison des 2 méthodes d'estimation}

Le comptage chromosomique par la méthode de Feulgen et la mesure des longueurs des cellules de garde des stomates ont été réalisés simultanément sur un échantillon de 50 plantes prises au hasard parmi la population de plantes d'origine pollinique.

Le comptage chromosomique effectué par la méthode de Feulgen montre que les 3 classes obtenues pour la longueur des cellules de garde correspondent respectivement aux niveaux haploïde, diploïde et triou polyploïde.

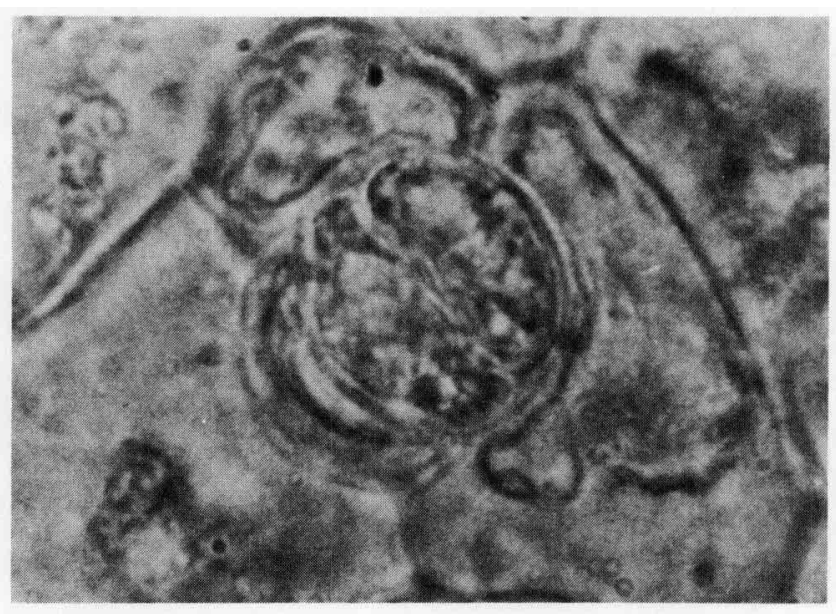

Figure 3

Stomate d'une plante haploïde.

Haploid plant stomata.

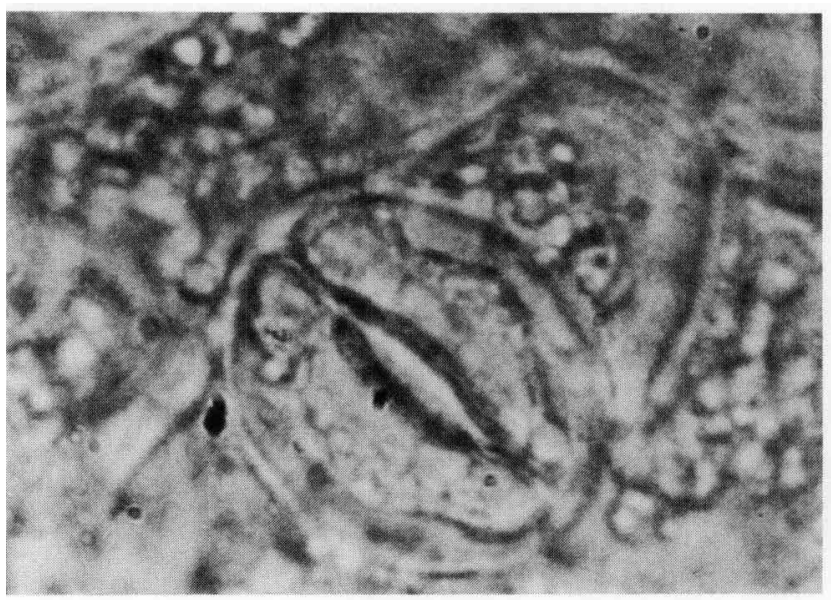

Figure 4

Stomate d'une plante diploïde.

Diploid plant stomata. 


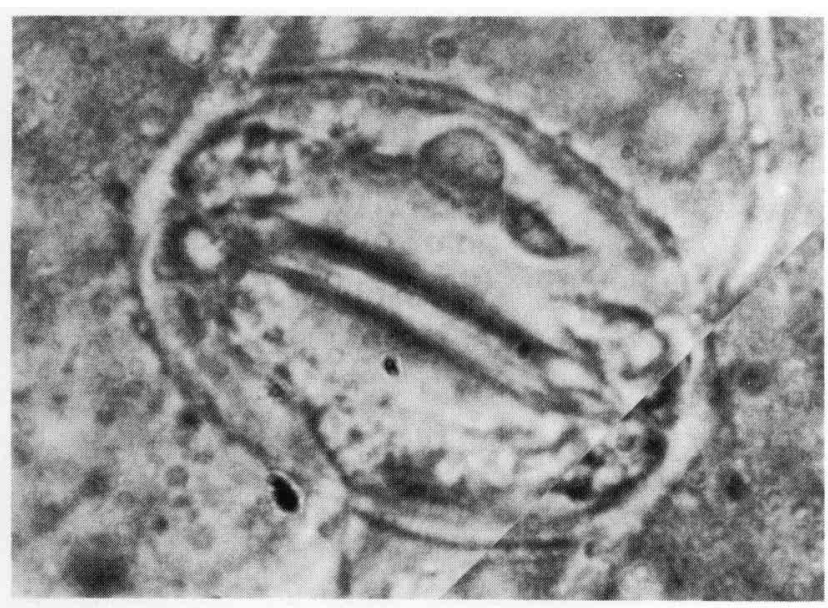

Figure 5

Stomate d'une plante triploïde.

Triploid plant stomala.

Les résultats obtenus entre les 2 méthodes sont en totale concordance dans plus de 80 p. 100 des cas (tabl. 1).

Ceci permet de considérer la méthode de mesure de la longueur des stomates comme un moyen efficace d'estimation du niveau de ploïdie et de l'adopter en remplacement de la méthode de comptage chromosomique.

Il est intéressant de signaler que les plantes dont les moyennes des mesures de la longueur des cellules de garde divergent d'une feuille à l'autre, s'avèrent mixoplö̈des. Ceci confïrme la nécessité d'observer au moins 2 feuilles par plante.

Dans l'échantillon des 50 plantes étudiées, les niveaux de ploïdie se répartissent comme suit :

- 23 p. 100 des plantes sont haploïdes,

- 64 p. 100 des plantes sont diploïdes,

- 13 p. 100 des plantes sont triploïdes, polyploïdes ou mixoploïdes.

\section{Utilisation de la méthode pour évaluer le niveau de ploïdie de la population}

La mesure de longueur des cellules de garde des stomates a été ensuite appliquée à l'ensemble de la population de plantes d'origine pollinique ce qui a permis de distinguer 20 p. 100 de plantes haploïdes, 73 p. 100 de plantes diploïdes et 7 p. 100 de plantes triploïdes ou polyploïdes.

Cette répartition n'est pas significativement différente de celle de l'échantillon qui a servi à comparer les 2 méthodes, ce qui permet de confirmer le bienfondé de son emploi. Il faut remarquer que cette répartition présente une forte majorité de plantes diploïdes, homozygotes dont le doublement du stock chromosomique a eu lieu spontanément, dès le premier stade du développement du gamétophyte in vitro.

Ce résultat est en accord avec ceux observés sur les Brassica issus de culture d'anthères, notamment par RENARD \& DOSBA (1980), OCKENDON (1984).

D'autre part, il est intéressant de constater a posteriori que les informations apportées par l'utilisation
TABLEAU 1

Comparaison des niveaux de ploidie obtenus à l'aide des deux méthodes d'estimation différentes. Comparison between ploidy levels obtained by' the two different estimation methods.

\begin{tabular}{cccc}
\hline $\begin{array}{c}\text { Niveau de } \\
\text { plö̈die }\end{array}$ & $\begin{array}{c}\text { Estimation par } \\
\text { comptage } \\
\text { chromosomique gueur des cellules }\end{array}$ & $\begin{array}{c}\text { Estimation par } \\
\text { mesure de }\end{array}$ & $\begin{array}{c}\text { Taux de } \\
\text { convergence } \\
(\%)\end{array}$ \\
\cline { 2 - 4 } Haploïde & 8 & 11 & 72,7 \\
\hline Diploïde & 31 & 30 & 96,8 \\
\hline $\begin{array}{c}\text { Triploìde ou } \\
\text { polyploïde } \\
\text { mixoploìde }\end{array}$ & 8 & 6 & 75,0 \\
\hline Total & 47 & 47 & 81,5 \\
\hline \hline
\end{tabular}

d'une seule feuille sont très fiables et permettent de déterminer le niveau de ploïdie avec un très faible risque d'erreur.

Sachant que la fréquence des chimères mixoploïdes est très faible, on peut donc envisager, dans le cadre d'un travail routinier de sélection, de n'employer qu'une seule feuille ce qui allègerait la quantité de mesures à effectuer ; l'emploi d'une $2^{\mathrm{e}}$ feuille étant seulement nécessaire lorsque les résultats obtenus ne sont pas assez discriminants.

\section{CONCLUSION}

Le niveau de ploïdie d'un nombre élevé de plantes peut être rapidement et efficacement évalué par la mesure de la longueur des cellules de garde des stomates.

Cette méthode apparaît donc chez le Chou de Bruxelles, comme une alternative opérationnelle au comptage chromosomique.

Des expériences plus récentes nous ont d'ailleurs montré qu'elle était applicable à d'autres types de Brassica oleracea telles que le Chou cabus et le Chou broccoli.

Il faut toutefois signaler que nous avons comparé ici 2 méthodes relatives d'estimation et que seule l'étape de la floraison de la plante permet de déterminer finalement le niveau de ploïdie réel du sporophyte.

D'un point de vue pratique, le repérage précoce des plantes haploïdes permet d'appliquer ensuite immédiatement un traitement à la plante pour effectuer le doublement du stock chromosomique.

Ce fait est très important car il rend finalement possible l'observation simultanée pour la sélection de l'ensemble des plantes homozygotes diploïdes, provenant du doublement du stock chromosomique d'un gamétophyte haploide aussi bien au stade embryonnaire in vitro qu'au stade de la plante entière en conditions horticoles. 


\section{REMERCIEMENTS}

Nous tenons à remercier Monsieur David OCKEnDON et Madame Yvonne CAUDERON pour leurs précieux conseils ainsi que Monsieur Lionel BOULIDARD qui nous a fourni le matériel végétal.

\section{RÉFÉRENCES BIBLIOGRAPHIQUES}

Cauderon Y., Cauderon A., 1953. Polyploïdie et polysomie chez Crepis zacintha (L.) Babc. Ann. Amélior. Plant., 4, 1-12.

Dunwell J. M., Cornish M., De Courcel A. G. L.., 1985. Influence of genotype, plant growth temperature and anther incubation temperature on microspore embryogenic production in Brassica napus ssp. oleifera. J. Exp. Bot., 36, 165, 679-689.

George L., Rao O. S., 1982. In vitro induction of pollen embryos and plantlets in Brassica juncea through anther culture. Plant Sci. Lett., 26, 1, 111-116.

Keller W., Armstrong K., 1977. Embryogenesis and plant regeneration in Brassica napus anther cultures. Can. J. Bot., 55, 1383-1388.

Keller W., Armstrong K., 1978. High frequency production of microspore-derived plants from Brassica napus anther culture. $Z$. Pflanzenzücht., 80, 100-108.

Keller W., Armstrong K., 1983. Production of haploids via anther culture in Brassica oleracea var. italica. Euphytica, 32, 151-199.

Lelu M. A., Bollon H., 1985. Obtention d'haploïdes par culture d'anthères de Brassica oleracea L. var. capitata et var. gemmifera. C.R. Acad. Sci, Paris, 300, 2, ser. 3, 71-76.

Ockendon D., 1984. Anther culture in Brussels sprouts (Brassica oleracea var. gemmifera). 1. Embryo yields and plant regeneration. Ann. appl. Biol., 105, 2, 285-291.

Ockendon D., Currah L., Robinson H. T., 1983. Anther culture of Brussels sprouts. Wellesbourne, Nat. Veg. Res. Sin. annu. Rep., p. 50 .

Orton T. J., Browers M. A., 1985. Segregation of genetic markers among plants regenerated from cultured anthers of broccoli (Brassica oleracea var. italica). Theor. appl. Genet. 69, 637-643.

Renard M., Dosba F., 1980. Etude de l'haploïdie chez le colza (Brassica napus L. var. oleifera Metzger). Ann. Amelior. Plant, 30, 2, 191-209.

Speckmann G. J., Post Jr. J., Dijkstra, 1965. The length of stomata as an indicator for polyploidy in rye-grasses. Euphytica, 14, 225-230. 